\title{
Commentary: Top-down and bottom-up modulation of pain-induced oscillations
}

\author{
Valentina Nicolardi ${ }^{1,2 *}$ and Elia Valentini ${ }^{3}$ \\ ${ }^{1}$ Department of Psychology, Sapienza University of Rome, Rome, Italy, ${ }^{2}$ Fondazione Santa Lucia, Istituto di Ricovero e Cura a \\ Carattere Scientifico, Rome, Italy, ${ }^{3}$ Department of Psychology and Centre for Brain Science, University of Essex, England, UK
}

Keywords: pain, attention, gamma-band, EEG, top-down modulation

\section{A commentary on}

Top-down and bottom-up modulation of pain-induced oscillations by Hauck, M., Domnick, C., Lorenz, J., Gerloff, C., and Engel, A. K. (2015). Front. Hum. Neurosci. 9:375. doi: 10.3389/fnhum.2015.00375

\section{OPEN ACCESS}

Edited by:

Klaus Gramann,

Berlin Institute of Technology,

Germany

Reviewed by:

Daniel Senkowski,

Charité - Universitätsmedizin Berlin,

Germany

Markus Ploner

Technische Universität München,

Germany

Michael Hauck,

University Hospital Hamburg

Eppendorf, Germany

*Correspondence:

Valentina Nicolardi

valentina.nicolardi@uniroma1.it

Received: 20 January 2016

Accepted: 28 March 2016

Published: 18 April 2016

Citation:

Nicolardi $V$ and Valentini $E$ (2016)

Commentary: Top-down and bottom-up modulation of pain-induced oscillations.

Front. Hum. Neurosci. 10:152. doi: 10.3389/fnhum.2016.00152
Attention represents one of the main mechanisms of modulation of pain (Wiech et al., 2008). It is a complex function which collects cognitive processes responsible for orienting to, and maintaining brain resources on, the most salient and relevant information for the ongoing behavioral demands (Knudsen, 2007). Whether attention can be captured by and oriented to sensory events depends on several variables, e.g., stimulus features, task demands, and volition (Baluch and Itti, 2011). Bottom-up capture of attention is often conceived as an unintentional and automatic process ruled by the competition between stimulus features, whereas top-down selection concerns an intentional process pertaining to intrinsic motivational and task-related aspects ruled by the subject (Buschman and Miller, 2007). Stimuli interpreted as painful are able to capture individual's attention in a bottom-up fashion if e.g., other stimuli or unrelated plans do not compete with its processing in working memory (Legrain et al., 2012).

In a recent issue of Frontiers in Human Neuroscience, Hauck et al. (2015) used electroencephalography (EEG) to investigate the oscillatory neuronal modulation exerted by both top-down and bottom-up attention during nociceptive painful stimulation. To disentangle how the two attentional processes influence neuronal synchronization and desynchronization, the authors manipulated both nociceptive stimulus intensity (i.e., high vs. low; bottom-up orientation) and spatial attention to the stimuli (i.e., attended vs. unattended; top-down control).

The authors already investigated the effect of spatial attention on cortical oscillatory activity elicited by electrical painful stimuli (Hauck et al., 2007) by recording magnetoencephalography (MEG) during an oddball paradigm whereby participants were asked to count the rare stimuli on one finger while ignoring the more frequent stimuli on the other finger. The authors concluded that increased attentional capture by salient rare stimuli was reflected by MEG-induced oscillatory high frequency activity (gamma band) localized in the sensory-motor areas. Hauck et al. (2015) expand on their previous methodology by varying location and intensity of laser painful stimuli. Here the participant had to attend and evaluate only stimuli delivered to one finger while ignoring those delivered to the other finger. At variance with their previous work, they delivered equiprobable stimuli on the ring and index finger, thus avoiding a bottom-up confounding effect associated with variable probability of occurrence of the stimuli.

They investigated attentional effects on the entire spectral activity and found that power (i.e., magnitude) of gamma oscillations increased (maximal at $270 \mathrm{~ms}$ post-stimulus) while alpha 
decreased $(750 \mathrm{~ms})$ during stimulation of the attended compared to the unattended finger. Conversely, the increase of stimulus intensity was associated with power increase in delta $(300 \mathrm{~ms})$ and gamma bands, and power decrease in alpha and beta bands $(600 \mathrm{~ms})$. Source analysis identified the contralateral insula as generator of gamma activity during top-down attentional control, and the sensory-motor and mid-cingulate regions as gamma activity generators during bottom-up capture of attention. The activation found in the sensory-motor areas during bottom-up attention is not surprising as previous studies reported the association between activity in the primary somatosensory cortex and selective nociceptive laser stimulation (Timmermann et al., 2001; Gross et al., 2007; Schulz et al., 2012; Valentini et al., 2012; Zhang et al., 2012).

A relevant aspect of Hauck et al.'s study (Hauck et al., 2015) is the differential modulation of gamma-band found during topdown and bottom-up attention to pain. To date, only Tiemann et al. (2015) explicitly investigated the functional significance of nociceptive-related gamma activity in these two conditions. They separately varied stimulus intensity, as a means to induce bottom-up modulation whereas they set out a placebo analgesia manipulation to induce the top-down modulation. Interestingly, while the design implemented by Tiemann et al. provides an orthogonal manipulation (i.e., intensity varies independently from cognitive expectation, and viceversa), the design devised by Hauck et al. did not manipulate the two variables separately, that is they required the participants to assess the intensity only for the stimuli delivered to the attended finger. Thus, they likely introduced a significant interaction between bottom-up and topdown processes in the "attended high" condition. Yet, their design does not allow to disambiguate this interaction.

Tiemann et al. (2015) found that nociceptive laser-evoked potentials were similarly influenced by both stimulus intensity and placebo analgesia whereas nociceptive-induced alpha and gamma responses were sensitive to changes in stimulus intensity but not to the induction of changes in beliefs about the experience of pain. Taking a closer look, one could argue that Hauck et al. (2015) may have enhanced the role of sensory features involved in the spatial localization of the stimuli compared to the placebo procedure implemented by Tiemann et al. (2015), which

\section{REFERENCES}

Baluch, F., and Itti, L. (2011). Mechanisms of top-down attention. Trends Neurosci. 34, 210-224. doi: 10.1016/j.tins.2011.02.003

Buschman, T. J., and Miller, E. K. (2007). Top-down versus bottom-up control of attention in the prefrontal and posterior parietal cortices. Science 315, 1860-1862. doi: 10.1126/science.1138071

Fries, P., Reynolds, J. H., Rorie, A. E., and Desimone, R. (2001). Modulation of oscillatory neuronal synchronization by selective visual attention. Science 291, 1560-1563. doi: 10.1126/science.1055465

Gross, J., Schnitzler, A., Timmermann, L., and Ploner, M. (2007). Gamma oscillations in human primary somatosensory cortex reflect pain perception. PLoS Biol. 5:e133. doi: 10.1371/journal.pbio.0050133

Hauck, M., Domnick, C., Lorenz, J., Gerloff, C., and Engel, A. K. (2015). Top-down and bottom-up modulation of pain-induced oscillations. Front. Hum. Neurosci. 9:375. doi: 10.3389/fnhum.2015.00375 more consistently triggered higher-level cortical activity over the sensory cortices (Wager et al., 2011).

Other studies reported a gamma-band modulation by topdown attention during different sensory modalities (Fries et al., 2001). Using a top-down attentional manipulation similar to Hauck et al. (2015) and Siegel et al. (2008) characterized the effect of attention on neuronal synchronization pattern across the human dorsal visual pathway. During the poststimulus interval they found higher gamma vs. lower alpha phase coherence between cortical regions involved in the processing of visual stimuli, and concluded that gamma band enhancement likely reflects local neuronal synchronization that is functional to the selective attention task. During subdural electrocorticography, Ray et al. (2008) investigated the effect of selectively attending one stimulus modality over the other in a crossmodal auditory and vibro-tactile stimulation. The authors found that gamma activity was greater over the auditory and somatosensory cortices when the auditory and tactile stimuli were attended. Overall, these studies support Hauck et al.'s (2015) interpretation that top-down selective attention is correlated with increased activity of gamma frequencies.

Despite differences in stimuli and methodology, altogether these studies indicate the gamma-band oscillatory activity may not only index bottom-up salience of the sensory event but also top-down relevance of the sensory representation for the ongoing behavioral goals. However, further methodological and analytical efforts will be needed to clarify the role of the gamma activity across different types of top-down attention manipulations.

\section{AUTHOR CONTRIBUTIONS}

VN has developed most of the content of the article. EV has supervised her during the writing and he has edited the text.

\section{ACKNOWLEDGMENTS}

This work was supported by a 2013 " 5 XMille" funds of The Italian Health Ministry to VN. The authors are grateful to Giuseppe Spinelli for his valuable comments.

Hauck, M., Lorenz, J., and Engel, A. K. (2007). Attention to painful stimulation enhances gamma-band activity and synchronization in human sensorimotor cortex. J. Neurosci. 27, 9270-9277. doi: 10.1523/JNEUROSCI.228307.2007

Knudsen, E. I. (2007). Fundamental components of attention. Annu. Rev. Neurosci. 30, 57-78. doi: 10.1146/annurev.neuro.30.051606.094256

Legrain, V., Mancini, F., Sambo, C. F., Torta, D. M., Ronga, I., and Valentini, E. (2012). Cognitive aspects of nociception and pain: bridging neurophysiology with cognitive psychology. Neurophysiol. Clin. 42, 325-336. doi: 10.1016/j.neucli.2012.06.003

Ray, S., Niebur, E., Hsiao, S. S., Sinai, A., and Crone, N. E. (2008). High-frequency gamma activity $(80-150 \mathrm{~Hz})$ is increased in human cortex during selective attention. Clin. Neurophysiol. 119, 116-133. doi: 10.1016/j.clinph.2007.09.136

Schulz, E., Tiemann, L., Witkovsky, V., Schmidt, P., and Ploner, M. (2012). gamma Oscillations are involved in the sensorimotor transformation of pain. J. Neurophysiol. 108, 1025-1031. doi: 10.1152/jn.00186.2012 
Siegel, M., Donner, T. H., Oostenveld, R., Fries, P., and Engel, A. K. (2008). Neuronal synchronization along the dorsal visual pathway reflects the focus of spatial attention. Neuron 60, 709-719. doi: 10.1016/j.neuron.2008.09.010

Tiemann, L., May, E. S., Postorino, M., Schulz, E., Nickel, M. M., Bingel, U., et al. (2015). Differential neurophysiological correlates of bottomup and top-down modulations of pain. Pain 156, 289-296. doi: 10.1097/01.j.pain.0000460309.94442.44

Timmermann, L., Ploner, M., Haucke, K., Schmitz, F., Baltissen, R., and Schnitzler, A. (2001). Differential coding of pain intensity in the human primary and secondary somatosensory cortex. J. Neurophysiol. 86, 1499-1503.

Valentini, E., Hu, L., Chakrabarti, B., Hu, Y., Aglioti, S. M., and Iannetti, G. D. (2012). The primary somatosensory cortex largely contributes to the early part of the cortical response elicited by nociceptive stimuli. Neuroimage 59, 1571-1581. doi: 10.1016/j.neuroimage.2011.08.069

Wager, T. D., Atlas, L. Y., Leotti, L. A., and Rilling, J. K. (2011). Predicting individual differences in placebo analgesia: contributions of brain activity during anticipation and pain experience. J. Neurosci. 31, 439-452. doi: 10.1523/JNEUROSCI.3420-10.2011
Wiech, K., Ploner, M., and Tracey, I. (2008). Neurocognitive aspects of pain perception. Trends Cogn. Sci. 12, 306-313. doi: 10.1016/j.tics.2008. 05.005

Zhang, Z. G., Hu, L., Hung, Y. S., Mouraux, A., and Iannetti, G. D. (2012). Gamma-band oscillations in the primary somatosensory cortex-a direct and obligatory correlate of subjective pain intensity. J. Neurosci. 32, 7429-7438. doi: 10.1523/JNEUROSCI.5877-11.2012

Conflict of Interest Statement: The authors declare that the research was conducted in the absence of any commercial or financial relationships that could be construed as a potential conflict of interest.

Copyright $\odot 2016$ Nicolardi and Valentini. This is an open-access article distributed under the terms of the Creative Commons Attribution License (CC BY). The use, distribution or reproduction in other forums is permitted, provided the original author(s) or licensor are credited and that the original publication in this journal is cited, in accordance with accepted academic practice. No use, distribution or reproduction is permitted which does not comply with these terms. 